PHYSICAL REVIEW D 93, 069904(E) (2016)

\title{
Erratum: Higgs effective potential in a perturbed Robertson-Walker background \\ [Phys. Rev. D 90, 123541 (2014)]
}

Antonio L. Maroto and Francisco Prada

(Received 24 February 2016; published 16 March 2016)

DOI: 10.1103/PhysRevD.93.069904

We correct an error in our previous computation of the one-loop contributions to the Higgs effective potential in a perturbed Friedmann-Robertson-Walker background. A term was missing in the next to leading order in the adiabatic expansion of the perturbed equations of motion. The mistake has propagated in the calculation of the divergences. Here we present the corrected results.

In our paper, the contribution from Higgs self-interactions to the one-loop effective potential in curved space-time was obtained using a comoving cutoff regularization. This method allowed us to obtain the divergent terms of the inhomogeneous contributions. In Ref. [1], the calculation was redone using dimensional regularization in order to compute also the finite parts of the integrals with a method which respects the symmetries of the classical theory.

In this process we identified a term missing in Eq. (42) of our paper corresponding to the next to leading order in the adiabatic approximation. The corrected equation now reads

$$
f_{k} \theta_{k}^{\prime \prime}+2 f_{k}^{\prime} \theta_{k}^{\prime}+\left[2 \mathcal{H}-\Phi^{\prime}-3 \Psi^{\prime}\right] f_{k} \theta_{k}^{\prime}-f_{k} \nabla^{2} \theta_{k}-2 \boldsymbol{\nabla} f_{k} \cdot \boldsymbol{\nabla} \theta_{k}-f_{k} \boldsymbol{\nabla} \theta_{k} \cdot \boldsymbol{\nabla}[\Phi-\Psi]=0 .
$$

This implies that Eq. (49) should read

$$
\begin{aligned}
& F_{k} \delta \theta_{k}^{\prime \prime}+2 F_{k}^{\prime} \delta \theta_{k}^{\prime}+2 \mathcal{H} F_{k} \delta \theta_{k}^{\prime}-F_{k} \nabla^{2} \delta \theta_{k}-2 \omega \delta f_{k}^{\prime}-2 \mathbf{k} \cdot \nabla \delta f_{k}-2 \omega \mathcal{H} \delta f_{k}-\omega^{\prime} \delta f_{k} \\
& \quad+\omega F_{k} \Phi^{\prime}+3 \omega F_{k} \Psi^{\prime}-F_{k} \mathbf{k} \cdot \nabla[\Phi-\Psi]=0 .
\end{aligned}
$$

Then Eq. (56) now reads

$$
H_{k}(\eta, \mathbf{p})=\omega\left(-i \frac{\alpha_{k}(\eta, \mathbf{p})}{\omega} \delta \theta_{k}(\eta, \mathbf{p})+\Psi(\eta, \mathbf{p})\left(3-\frac{k^{2}}{\omega^{2}}\right)\right)^{\prime}+p^{2} \delta \theta_{k}(\eta, \mathbf{p})-i \alpha_{k}(\eta, \mathbf{p})(\Phi(\eta, \mathbf{p})-\Psi(\eta, \mathbf{p})) \omega .
$$

The new contribution implies that the cutoff expansion of the inhomogeneous contribution Eq. (68) is

$$
V_{\mathrm{eff}}^{i}(\hat{\phi})=\frac{m^{2}(\hat{\phi}) \Lambda^{2}}{8 \pi^{2} a^{2}} \mathcal{F}\left[\frac{\sin (p \eta)}{p \eta}(\Phi+\Psi)-\Phi\right]+\mathcal{O}\left(\Lambda^{-2}\right)
$$

In other words, the logarithmic divergences exactly vanish in agreement with the results in dimensional regularization in Ref. [1]. As already discussed in our paper, the quadratic divergence required the introduction of new counterterms which were not present in the original Lagrangian. This problem is also solved when considering dimensional regularization since in that case the inhomogeneous contribution is finite.

The analysis on the variations of the Higgs vacuum expectation value in our paper based on the logarithmic contribution therefore does not apply. The corrected analysis based on the finite contributions can be found in Ref. [2].

\section{ACKNOWLEDGMENTS}

This work has been supported by the Spanish MICINN Consolider-Ingenio 2010 Programme under Grant MultiDark CSD2009-0006 and MINECO Grants No. FIS2014-52837-P, No. AYA-2012-31101 and No. AYA2014-60641-C2-1-P.

[1] F. D. Albareti, A. L. Maroto, and F. Prada, Gravitational perturbations of the Higgs field, arXiv:1602.02776.

[2] F. D. Albareti, A. L. Maroto, and F. Prada, Gravitational effects on the Higgs field within the Solar System, arXiv:1602.03290. 